\title{
A 10-Week Memantine Treatment in Bipolar Depression: A Case Report. Focus on Depressive Symptomatology, Cognitive Parameters and Quality of Life
}

\author{
Dominik Strzelecki ${ }^{1}$, Agnieszka Tabaszewska², Zbigniew Barszcz ${ }^{2}$, \\ Olga Józefowicz ${ }^{1}$, Paweł Kropiwnicki ${ }^{3}$, and Jolanta Rabe-Jabłońska ${ }^{1}$ \\ ${ }^{1}$ Department of Affective and Psychotic Disorders, Medical University of Łódź, Łódź, Poland \\ ${ }^{2}$ Central Clinical Hospital, Łódź, Poland \\ ${ }^{3}$ Department of Adolescent Psychiatry, Medical University of Łódź, Łódź, Poland
}

\begin{abstract}
Memantine and other glutamatergic agents have been currently investigated in some off-label indications due to glutamatergic involvement in several psychoneurological disorders. We assumed that memantine similarly to ketamine may positively influence mood, moreover having a potential to improve cognition and general quality of life. We report a case of a 49-year-old male hospitalized during a manic and a subsequent moderate depressive episode. After an ineffective use of lithium, olanzapine and antidepressive treatment with mianserin, memantine was added up to $20 \mathrm{mg}$ per day for 10 weeks. The mental state was assessed using the Hamilton Depression Rating Scale, the Young Mania Rating Scale, the Hamilton Anxiety Scale, the Clinical Global Inventory, the World Health Organization Quality of Life Scale and psychological tests. After 10 weeks the patient achieved a partial symptomatic improvement in mood, anxiety and quality of sleep, but his activity remained insufficient. We also observed an improvement in the parameters of cognitive functioning and quality of life. There was neither significant mood variations during the memantine use nor mood changes after its termination. No significant side effects were noted during the memantine treatment. We conclude that using memantine in bipolar depression may improve mood, cognitive functioning and quality of life.

Psychiatry Investig 2013;10:421-424
\end{abstract}

Key Words Memantine, Bipolar disorder, Depression, Cognitive functioning, Glutamate.

\section{INTRODUCTION}

Glutamatergic system is the largest and most important excitatory system of the human brain and plays an important, if not crucial role in the pathogenesis of numerous mental and neurological disorders, such as schizophrenia, Alzheimer's disease and alcohol dependency. ${ }^{1,2}$

The effects of glutamatergic modulation have also been studied in depression and bipolar disorder (BD) - lamotrigine, lithium or valproate (mood stabilizers) and riluzole were proven to have properties to modulate glutamatergic transmission. ${ }^{3,4}$ The effects of ketamine, a potent glutamatergic NMDA

Received: September 27, 2012 Revised: January 8, 2013

Accepted: January 20, 2013 Available online: December 16, 2013

$\triangle$ Correspondence: Dominik Strzelecki

Department of Affective and Psychotic Disorders, Medical University of Łódź, ul. Pomorska 251, 92-213 Łódź, Poland

Tel: +48426757236 , Fax: +48426757403

E-mail: dominik.strzelecki@umed.lodz.pl

(a) This is an Open Access article distributed under the terms of the Creative Commons Attribution Non-Commercial License (http://creativecommons.org/licenses/by$\mathrm{nc} / 3.0$ ) which permits unrestricted non-commercial use, distribution, and reproduction in any medium, provided the original work is properly cited. receptor antagonist are particularly interesting and clinically important in the treatment of affective disorders - after a single dose of ketamine depressive patients attained significant improvement within hours after injection. ${ }^{5}$ Its clinical use is limited because ketamine requires intravenous administration and many hours of observation due to the risk of serious side effects like hypertension and psychotic symptoms.

The drug significantly better than ketamine should be similarly effective but better tolerated, and suitable for oral administration and long term treatment. Memantine preliminarily seems to meet most of these conditions, it is a much easier-inuse and a safer therapeutic option than ketamine, but its efficacy should be precisely evaluated.

Memantine is approved for the treatment of moderate to severe forms of Alzheimer's disease being a non-competitive NMDA receptor antagonist with a medium affinity, characterized by a rapid mechanism of switching. By binding a receptor inside the ion channel, memantine inhibits the flow of $\mathrm{Ca}^{2+}$ (important e.g. for memory or excitatory damage processes), $\mathrm{Na}^{+}$and $\mathrm{K}^{+}$ions. Hyperactivity of the glutamate system and 
the NMDA receptor leads to excitotoxicity and dementia. Normalization of glutamatergic transmission with memantine improves cognition and slows the neurodegenerative processes. ${ }^{6-9}$ Hypothetic mechanisms of memantine action in affective disorders are not entirely clear, but reduction of glutamatergic transmission and normalization of the NMDA-dependent $\mathrm{Na}^{+}$ and $\mathrm{Ca}^{2+}$ currents may both stabilize and improve the mood. ${ }^{10}$ Although there have been only a few clinical studies of memantine in affective disorders (mostly in unipolar depression and a treatment-resistant bipolar disorder), anxiety disorders (promising results in obsessive-compulsive disorder and posttraumatic stress disorder) and schizophrenia, the preliminary results show some benefits. ${ }^{11-25}$ Our case report is one of the first works describing the impact of memantine on mood and the first one describing cognitive functions and quality of life changes during memantine use in a bipolar depressive episode. Moreover, this is the first case report indicating that memantine may more effectively improve the mood than an antidepressive agent - mianserin. Our project was accepted by the Ethics Committee of Medical University of Łódź (RNN/ 233/12/KE).

\section{CASE}

A 49-year-old male with the diagnosis of type I BD, treated psychiatrically for over 20 years, previously 5 times hospitalized, was admitted in a manic episode, when he tried to force the hospital door. During the first 2 weeks of olanzapine treatment the manic symptoms have been reduced. After the manic episode ended, some depressive symptoms emerged, quickly reaching a moderate severity. Continuing the therapy with lithium (1 g/day) and olanzapine (7.5 mg/day) for several weeks we have not noted any significant improvement. Treatment with mianserin, an alpha adrenergic antidepressant charac- terized by a low switch risk was started. After 6 weeks of mianserin (initially at $30 \mathrm{mg} /$ day for 3 weeks, then raised to 60 $\mathrm{mg}$ /day) there was still no clinical improvement. After obtaining an informed consent, the patient started the intake of memantine with no other changes of the treatment. For the first week the daily memantine dose was $5 \mathrm{mg}$, every week it was increased by $5 \mathrm{mg}$ to a maximum of $20 \mathrm{mg}$. Tolerance of the drug throughout its use was very good, the patient did not report any side effects whatsoever.

The patient had no other chronic illness than BD. Neurological assessments showed no abnormalities; the hematological, biochemical and hormonal (TSH) parameters were normal. In the CT scan no significant morphological changes were found.

Every week during the10 weeks of memantine treatment and also 6 weeks after its termination the patient was examined using the Hamilton Depression Rating Scale (HDRS), Young Mania Rating Scale (YMRS), Hamilton Anxiety Scale (HAMA), Clinical Global Impression (CGI) and World Health Organization Quality of Life Scale (WHOQOL-BREF short version, Table 1). Before starting memantine (week 0W0) and at the end of its use (W10) the patient also performed psychological examination panel (Table 2).

\section{Psychiatric symptoms}

After the memantine initiation we observed a consecutive improvement of depressive symptoms, which was noted early, what is congruent with the observations of Anand et al. ${ }^{13}$ We noted a significant improvement in mood, sleep parameters and appetite. After a quick improvement following first doses of memantine the level of anxiety remained on the same mild level. During the memantine period a brief mild depressive exacerbation was observed (ca. W5), which was conditioned by objective external circumstances, but with this exception the

Table 1. Psychiatric assessments results

\begin{tabular}{|c|c|c|c|c|c|c|c|c|c|c|c|c|c|c|c|c|}
\hline & W0 & W1 & W2 & W3 & W4 & W5 & W6 & W7 & W8 & W9 & W10 & W11 & W12 & W13 & W14 & W15 \\
\hline HDRS & 19 & 15 & 13 & 11 & 11 & 16 & 13 & 12 & 11 & 11 & 11 & 9 & 10 & 9 & 6 & 4 \\
\hline YMRS & 0 & 0 & 0 & 0 & 2 & 0 & 2 & 2 & 0 & 0 & 0 & - & - & - & - & 0 \\
\hline HAMA & 15 & 8 & 7 & 6 & 8 & 8 & 9 & 9 & 8 & 7 & 7 & - & - & - & - & 3 \\
\hline CGI & 4 & 3 & 3 & 3 & 3 & 4 & 3 & 3 & 3 & 3 & 3 & 3 & 3 & 3 & 2 & 2 \\
\hline CGI-I & 3 & 3 & 3 & 3 & 3 & 4 & 3 & 3 & 3 & 3 & 3 & 3 & 3 & 3 & 2 & 2 \\
\hline WHOQOL1 & 17 & 19 & 19 & 20 & 20 & 18 & 19 & 18 & 18 & 17 & 17 & - & - & - & - & 21 \\
\hline WHOQOL2 & 17 & 15 & 15 & 15 & 15 & 14 & 15 & 15 & 15 & 16 & 17 & - & - & - & - & 19 \\
\hline WHOQOL3 & 7 & 7 & 7 & 7 & 7 & 7 & 8 & 7 & 7 & 8 & 8 & - & - & - & - & 8 \\
\hline WHOQOL4 & 19 & 22 & 20 & 26 & 25 & 25 & 24 & 24 & 24 & 24 & 23 & - & - & - & - & 27 \\
\hline WHOQOL & 60 & 63 & 61 & 68 & 67 & 64 & 66 & 64 & 64 & 65 & 65 & - & - & - & - & 75 \\
\hline
\end{tabular}

Abbreviations not used in the text: W0-W15: weeks 0-15, CGI-I: Clinical Global Impression-Improvement, WHOQOL parts: 1 (somatic), 2 (psychologic), 3 (social), 4 (environment) and total score. HDRS: Hamilton Depression Rating Scale, YMRS: Young Mania Rating Scale, HAMA: Hamilton Anxiety Scale, CGI: Clinical Global Impression, WHOQOL: World He-alth Organization Quality of Life Scale 
Table 2. Psychological test results

\begin{tabular}{lcc}
\hline & W0 & W10 \\
\hline WCST errors (\%) & 23 & 26 \\
WCST pers err (\%) & 8 & 13 \\
WCST n-pers err (\%) & 14 & 14 \\
WCST cat (\%) & 6 & 35, no errors \\
TMT1 (s) & 34, no errors & 114 , no errors \\
TMT2 (s) & 196, numerous errors & 23 items \\
Semantic verbal fluency & 19 elements, 1 perseveration & 11 elements \\
Phonemic verbal fluency & 8 elements+perseverations & Q2, Q2, Q4e \\
d2 test & Q1, Q1, Q4 & $4,10,9,11,11$ items \\
AVLT & $2,5,5,5,5$ items & 8 items \\
1. Learning curve & 4 items+1 confabulation & 3 errors \\
2. Recall & 5 errors & 10 items \\
3. Recognition & 4 items & \\
4. Delayed recall & &
\end{tabular}

pers err: perseverative errors, n-pers err: nonperseverative errors, cat: number of categories, WCST: Wisconsin Card Sorting Test, TMT: Trail Making Test, AVLT: Auditory-Visual Learning Test

mood was quite stable and the patient gradually improved.

\section{Psychological assessments (Table 2)}

In depressive, hypomanic or manic but also euthymic bipolar patients the cognitive functioning is significantly worse than in normal population. ${ }^{22,23}$ Assessing the progress in the treatment we compared the performance results of such functions as attention [Wisconsin Card Sorting Test (WCST), d2 test], direct visual memory [Benton Visual Retention Test (BVRT) - method A, versions C and D], direct auditory memory (repeating digits directly) and operational memory (WCST, repeating digits backwards), visuo-spatial working memory [WCST, Trail Making Test (TMT) - Part B], psychomotor speed (TMT - Part A), language and communication functions of the right hemisphere [The Right Hemisphere Language Battery, polish version (RHLB-PL)], auditory verbal learning [Auditory-Visual Learning Test (AVLT) - with two alternative versions], verbal fluency (semantic: animals, fruits and phonemic: letters K and S) and executive functions [Ruff Figural Fluency Test (RFFT), TMT - Part B].

Cognitive functioning results before and after the treatment indicate a marked improvement in auditory verbal learning, accuracy of verbal material learning and reduction of susceptibility to distraction. The numerical parameters of concentration, perceptual speed and accuracy of work also improved.

The overall result of right hemisphere language functions and communication skills, direct auditory and operating memory and psychomotor speed remained on similar levels before and after the memantine treatment.

The results of direct visual memory tests are difficult to comment. The numerical parameters (the number of wrong and right answers) were slightly worse in the second assessment, however, despite the alternative version of the test, it cannot be regarded as equivalent in terms of psychometric properties. The WCST results were slightly worse on the second assessment.

The numerical parameters (both semantic and phonemic) of verbal fluency in both assessments were similar.

In the RFFT and TMT (Part B) tests, assessing - among others - executive functions, a qualitative improvement in the efficiency of planning (the use of a rotation strategy in RFFT) and also in the control of action (error-free switching of the TMT) was observed. This improvement may be partly explained by the learning effect.

\section{Quality of life}

Quality of life was assessed using the popular WHOQOLBREF (Table 1). Parallelly to the clinical improvement, we also noted better quality of life parameters in the items describing physical functioning - e.g., sleep, energy, treatment, pain (part 1) and environment - finances, health services, living place (part 4).

Due to persistent mild depressive symptoms with a relatively excessive drive reduction, memantine and mianserin were stopped and venlafaxine was started (up to $75 \mathrm{mg}$ per day). This resulted in a satisfactory drive improvement and remission of the depression without any manic symptoms for 3 weeks (weeks 11-15 in Table 2).

\section{DISCUSSION}

Understanding the involvement of the glutamatergic system in the control of mood may lead to the intensification of research in this field and finding new therapeutic options in 
$\mathrm{BD}$ and major depression. Modulation of glutamatergic metabotropic and ionotropic AMPA receptors is considered a promising therapeutic approach in the treatment of affective disorders. $^{26,27}$

In the described patient with bipolar depression we observed a symptomatic improvement during the memantine augmentation of lithium, olanzapine and mianserin treatment. Interestingly, memantine was significantly more effective comparing to mianserin. We do not consider that the observed improvement is linked with the natural end of the depressive period because part of the important symptoms (e.g., drive reduction, decreased activity) remained on similar levels during the entire observation period. However, improvement in other basic depressive symptoms as mood, quality of sleep, appetite and also stability of mood was clinically relevant indicating the efficacy of memantine. Due to the methodological limitations of case reports conclusions should be formulated carefully.

Considering the cognitive parameters, after 10 weeks of memantine use we observed a mild improvement in most aspects of functioning. Clearer improvement resulting from the action of memantine and lower severity of depressive symptoms would be expected, however even in euthymic bipolar patients cognition dysfunction in most aspects is similar to that in mild cognitive impairment (MCI). ${ }^{23}$ In this relatively short observation period there were no major mood swings. This may indicate some mood stabilizing properties of memantine. Also the general quality of life of the patient has improved. Very good tolerability of memantine should also be emphasized here.

Further clinical trials of memantine and other glutamatergic agents assessing its antidepressive, antimanic and/or mood stabilizing properties are needed.

\section{REFERENCES}

1. Tuominen HJ, Tiihonen J, Wahlbeck K. Glutamatergic drugs for schizophrenia. Cochrane Database Syst Rev 2006;19:CD003730.

2. Nagy J. Alcohol related changes in regulation of NMDA receptor functions. Curr Neuropharmacol 2008;6:39-54.

3. Pittenger C, Coric V, Banasr M, Bloch M, Krystal JH, Sanacora G. Riluzole in the treatment of mood and anxiety disorders. CNS Drugs 2008; 22:761-786

4. Larkin GL, Beautrais AL. A preliminary naturalistic study of low-dose ketamine for depression and suicide ideation in the emergency department. Int J Neuropsychopharmacol 2011;14:1127-1131.

5. Zajączkowski W, Frankiewicz T, Parsons CG, Danysz W. Uncompetitive NMDA receptor antagonists attenuate NMDA-induced impairment of passive avoidance learning and LTP. Neuropharmacology 1997;36:961971.

6. Parsons CG, Danysz W, Quack G. Memantine is a clinically well tolerated N-methyl-D-apartate (NMDA) receptor antagonist - a review of preclinical data. Neuropharmacology 1999;38:735-767.

7. Standridge JB. Pharmacotherapeutic approaches to the treatment of Alzheimer's disease. Clin Ther 2004;26:615-630.

8. Ebixa (Memantine) Monograph. Available at: http://pl.lundbeck.com/
pl/PDF/Ebixa\%20SA/EBX_Monograph.pdf. Accessed September 14, 2012.

9. Permoda-Osip A, Rybakowski J. Glutamatergic conception of mood disorders. Psychiatr Pol 2011;45: 875-888.

10. Zarate CA Jr, Payne JL, Quiroz J, Sporn J, Denicoff KK, Luckenbaugh D, et al. An open-label trial of riluzole in patients with treatment-resistant major depression. Am J Psychiatry 2004;161:171-174.

11. Ferguson JM, Shingleton RN. An open-label, flexible-dose study of memantine in major depressive disorder. Clin Neuropharmacol 2007;30: 136-144.

12. Koukopoulos A, Serra G, Koukopoulos AE, Reginaldi D, Serra G. The sustained mood-stabilizing effect of memantine in the management of treatment resistant bipolar disorders: findings from a 12-month naturalistic trial. J Affect Disord 2012;136:163-166.

13. Anand A, Gunn AD, Barkay G, Karne HS, Nurnberger JI, Mathew SJ, et al. Early antidepressant effect of memantine during augmentation of lamotrigine inadequate response in bipolar depression: a double-blind, randomized, placebo-controlled trial. Bipolar Disord 2012;14:64-70.

14. Keck PE Jr, Hsu HA, Papadakis K, Russo J Jr. Memantine efficacy and safety in patients with acute mania associated with bipolar I disorder: a pilot evaluation. Clin Neuropharmacol 2009;32:199-204.

15. Muhonen LH, Lönnqvist J, Juva K, Alho H. Double-blind, randomized comparison of memantine and escitalopram for the treatment of major depressive disorder comorbid with alcohol dependence. J Clin Psychiatry 2008;69:392-399.

16. Muhonen LH, Lahti J, Sinclair D, Lönnqvist J, Alho H. Treatment of alcohol dependence in patients with co-morbid major depressive disorder -- predictors for the outcomes with memantine and escitalopram medication. Subst Abuse Treat Prev Policy 2008;3:20.

17. Teng CT, Demetrio FN. Memantine may acutely improve cognition and have a mood stabilizing effect in treatment-resistant bipolar disorder. Rev Bras Psiquiatr 2006;28:252-254.

18. Munoz C, Yulan N, Achaval V, Appiani F, Carroll BT. Memantine in major depression with catatonic features. J Neuropsychiatry Clin Neurosci 2008;20:119-120

19. Obregon DF, Velasco RM, Wuerz TP, Catalano MC, Catalano G, Kahn D. Memantine and catatonia: a case report and literature review. J Psychiatr Pract 2011;17:292-299.

20. Northoff G. What catatonia can tell us about "top-down modulation": a neuropsychiatric hypothesis. Behav Brain Sci 2002;25:555-577.

21. Agarwal V, Tripathi A. Memantine in the management of a clinically challenging case of bipolar disorder. Indian J Psychiatry 2009;51:137138.

22. Martínez-Arán A, Vieta E, Reinares M, Colom F, Torrent C, SánchezMoreno J, et al. Cognitive function across manic or hypomanic, depressed, and euthymic states in bipolar disorder. Am J Psychiatry 2004; 161:262-270.

23. Osher Y, Dobron A, Belmaker RH, Bersudsky Y, Dwolatzky T. Computerized testing of neurocognitive function in euthymic bipolar patients compared to those with mild cognitive impairment and cognitively healthy controls. Psychother Psychosom 2011;80:298-303.

24. Sani G, Serra G, Kotzalidis GD, Romano S, Tamorri SM, Manfredi G, et al. The role of memantine in the treatment of psychiatric disorders other than the dementias: a review of current preclinical and clinical evidence. CNS Drugs 2012;26:663-690.

25. Koukopoulos A, Reginaldi D, Serra G, Koukopoulos A, Sani G, Serra G. Antimanic and mood-stabilizing effect of memantine as an augmenting agent in treatment-resistant bipolar disorder. Bipolar Disord 2010; 12:348-349.

26. Witkin JM, Li X. New approaches to the pharmacological management of major depressive disorder. Adv Pharmacol 2009;57:347-379.

27. Enz R. Metabotropic glutamate receptors and interacting proteins: evolving drug targets. Curr Drug Targets 2012;13:145-156. 